\title{
Evaluating newly approved drugs for multidrug-resistant tuberculosis (endTB): study protocol for an adaptive, multi- country randomized controlled trial
}

L. Guglielmetti ${ }^{1,2,3}$, E. Ardizzoni ${ }^{4}$, M. Atger ${ }^{1}$, E. Baudin ${ }^{5}$, E. Berikova ${ }^{6,7}$, M. Bonnet ${ }^{1,8}$, E. Chang ${ }^{9}$, S. Cloez ${ }^{1}$, J. M. Coit ${ }^{10}$, V. Cox ${ }^{11}$, B. C. de Jong ${ }^{4}$, C. Delifer ${ }^{1}$, J. M. Do ${ }^{10}$, D. Dos Santos Tozzi ${ }^{5}$, V. Ducher ${ }^{1}$, G. Ferlazzo ${ }^{12}$, M. Gouillou' A. Khan ${ }^{13}$, U. Khan ${ }^{13}$, N. Lachenal ${ }^{1}$, A. N. LaHood ${ }^{10}$, L. Lecca ${ }^{10,14}$, M. Mazmanian ${ }^{1,15}$, H. Mcllleron ${ }^{16,17}$, M. Moschioni ${ }^{1}$, K. O'Brien ${ }^{18}$, O. Okunbor ${ }^{19}$, L. Oyewusi ${ }^{20}$, S. Panda ${ }^{21,22}$, S. B. Patil ${ }^{22}$, P. P. J. Phillips ${ }^{23}$, L. Pichon ${ }^{1}$, P. Rupasinghe ${ }^{4}$, M. L. Rich ${ }^{10,24,25}$, N. Saluhuddin ${ }^{26}$, K. J. Seung ${ }^{10,24,25}$, M. Tamirat ${ }^{20}$, L. Trippa ${ }^{27,28}$, M. Cellamare ${ }^{27}$, G. E. Velásquez ${ }^{10,25,29}$, S. Wasserman ${ }^{30,31}$, P. J. Zimetbaum ${ }^{32,33}$, F. Varaine ${ }^{1 \dagger}$ and C. D. Mitnick ${ }^{10,24,25^{*+}}$ (D)

\begin{abstract}
Background: Treatment of multidrug- and rifampin-resistant tuberculosis (MDR/RR-TB) is expensive, labourintensive, and associated with substantial adverse events and poor outcomes. While most MDR/RR-TB patients do not receive treatment, many who do are treated for 18 months or more. A shorter all-oral regimen is currently recommended for only a sub-set of MDR/RR-TB. Its use is only conditionally recommended because of very lowquality evidence underpinning the recommendation. Novel combinations of newer and repurposed drugs bring hope in the fight against MDR/RR-TB, but their use has not been optimized in all-oral, shorter regimens. This has greatly limited their impact on the burden of disease. There is, therefore, dire need for high-quality evidence on the performance of new, shortened, injectable-sparing regimens for MDR-TB which can be adapted to individual patients and different settings.
\end{abstract}

\footnotetext{
* Correspondence: carole mitnick@hms.harvard.edu

${ }^{\dagger} \mathrm{F}$. Varaine and C. D. Mitnick contributed equally to this work.

${ }^{10}$ Department of Global Health and Social Medicine, Harvard Medical School,

Boston, MA, USA

${ }^{24}$ Partners In Health, Boston, MA, USA

Full list of author information is available at the end of the article
}

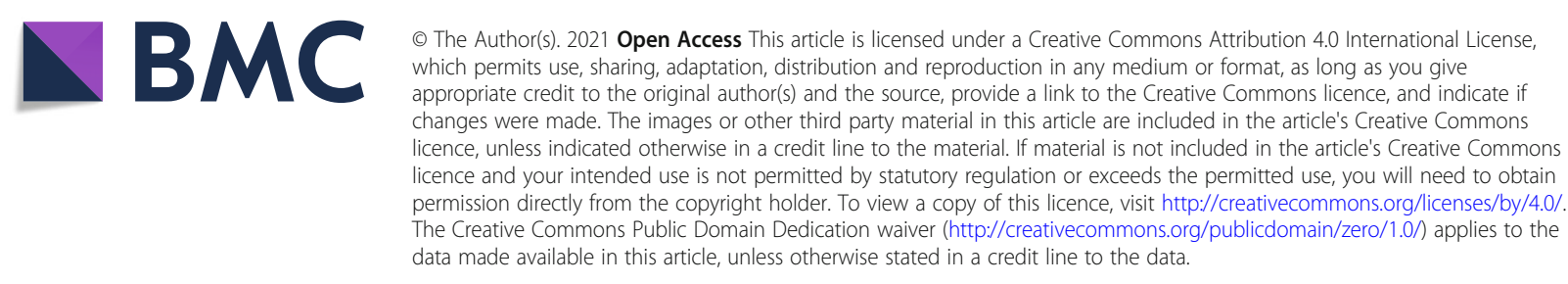


Methods: endTB is a phase III, pragmatic, multi-country, adaptive, randomized, controlled, parallel, open-label clinical trial evaluating the efficacy and safety of shorter treatment regimens containing new drugs for patients with fluoroquinolone-susceptible, rifampin-resistant tuberculosis. Study participants are randomized to either the control arm, based on the current standard of care for MDR/RR-TB, or to one of five 39-week multi-drug regimens containing newly approved and repurposed drugs. Study participation in all arms lasts at least 73 and up to 104 weeks post-randomization. Randomization is response-adapted using interim Bayesian analysis of efficacy endpoints. The primary objective is to assess whether the efficacy of experimental regimens at 73 weeks is noninferior to that of the control. A sample size of 750 patients across 6 arms affords at least $80 \%$ power to detect the non-inferiority of at least 1 (and up to 3) experimental regimens, with a one-sided alpha of 0.025 and a noninferiority margin of 12\%, against the control in both modified intention-to-treat and per protocol populations.

Discussion: The lack of a safe and effective regimen that can be used in all patients is a major obstacle to delivering appropriate treatment to all patients with active MDR/RR-TB. Identifying multiple shorter, safe, and effective regimens has the potential to greatly reduce the burden of this deadly disease worldwide.

Trial registration: ClinicalTrials.gov Identifier NCT02754765. Registered on 28 April 2016; the record was last updated for study protocol version 3.3, on 27 August 2019.

Keywords: Rifampin-resistant tuberculosis, Rifampicin-resistant tuberculosis, Bedaquiline, Delamanid, Linezolid, Clofazimine, Fluoroquinolone, Pyrazinamide, Treatment shortening, MDR-TB, Non-inferiority, Bayesian adaptive randomization

\section{Administrative information}

Note: the numbers in curly brackets in this protocol refer to SPIRIT checklist item numbers. The order of the items has been modified to group similar items (see http://www.equator-network.org/reporting-guidelines/ spirit-2013-statement-defining-standard-protocol-itemsfor-clinical-trials/).

\begin{tabular}{|c|c|}
\hline Title $\{1\}$ & $\begin{array}{l}\text { Evaluating newly approved drugs } \\
\text { for multidrug-resistant tuberculosis } \\
\text { (endTB): study protocol for an adap- } \\
\text { tive, multi-country randomized con- } \\
\text { trolled trial }\end{array}$ \\
\hline
\end{tabular}

Trial registration $\{2 \mathrm{a}$ and $2 \mathrm{~b}\} . \quad$ The endTB trial (evaluating newly approved drugs for multidrug-resistant tuberculosis, clinicaltrials.gov NCT02754765)

Protocol version $\{3\}$

Funding $\{4\}$
The clinicaltrials.gov record was last updated for study protocol version 3.3, on August 27, 2019

The trial and the preparation of this manuscript are funded by a grant provided by Unitaid. The funding source had no role in the study design; in the collection, management, analysis, or interpretation of data; in the writing of the report; or in the decision to submit the report for publication. GEV received funding from the National Institute of Allergy and Infectious Diseases at the U.S. National Institutes of Health (grant numbers K08 Al141740, L30 Al120170, and P30 Al060354), the Dr. Lynne Reid/Drs. Eleanor and Miles Shore Fellowship at Harvard Medical School, the Burke Global Health Fellowship at the Harvard Global Health Institute, and the Harvard University Center for AIDS Research.

\section{Administrative information (Continued)}

\begin{tabular}{ll} 
Title \{1\} & Evaluating newly approved drugs \\
for multidrug-resistant tuberculosis \\
(endTB): study protocol for an adap- \\
tive, multi-country randomized con- \\
trolled trial \\
\hline Médecins Sans Frontières, Paris, \\
France. \\
2 Sorbonne Université, INSERM, U1135, \\
Centre d'Immunologie Et Des Maladies \\
Infectieuses, Paris, France. \\
${ }^{3}$ Assistance Publique Hôpitaux de Paris \\
(APHP), Groupe Hospitalier Universitaire \\
Sorbonne Université, Hôpital Pitié- \\
Salpêtrière, Centre National De \\
Référence Des Mycobactéries Et De La \\
Résistance Des Mycobactéries Aux \\
Antituberculeux, Paris, France. \\
4 Institute of Tropical Medicine (ITM), \\
Antwerp, Belgium. \\
5 Epicentre, Paris, France. \\
6 Partners In Health, Astana, Kazakhstan. \\
7 National Scientific Center of \\
Phthisiopulmonology, Almaty, \\
Kazakhstan. \\
8 IRD UMI233/ INSERM U1175/Université \\
de Montpellier, Montpellier, France. \\
9 Médecins Sans Frontières, Toronto, \\
Ontario. \\
10 Department of Global Health and \\
Social Medicine, Harvard Medical \\
School, Boston, Massachusetts. \\
11 Centre for Infectious Disease \\
Epidemiology and Research (CIDER), \\
School of Public Health and Medicine, \\
Faculty of Health Sciences, University of \\
Cape Town, South Africa. \\
12 Southern Africa Medical Unit, \\
Médecins Sans Frontières, Cape Town, \\
South Africa. \\
13 Interactive Research and \\
Development, Karachi, Pakistan. \\
\end{tabular}




\section{Administrative information (Continued)}

\begin{tabular}{|c|c|}
\hline Title $\{1\}$ & $\begin{array}{l}\text { Evaluating newly approved drugs } \\
\text { for multidrug-resistant tuberculosis } \\
\text { (endTB): study protocol for an adap- } \\
\text { tive, multi-country randomized con- } \\
\text { trolled trial }\end{array}$ \\
\hline & 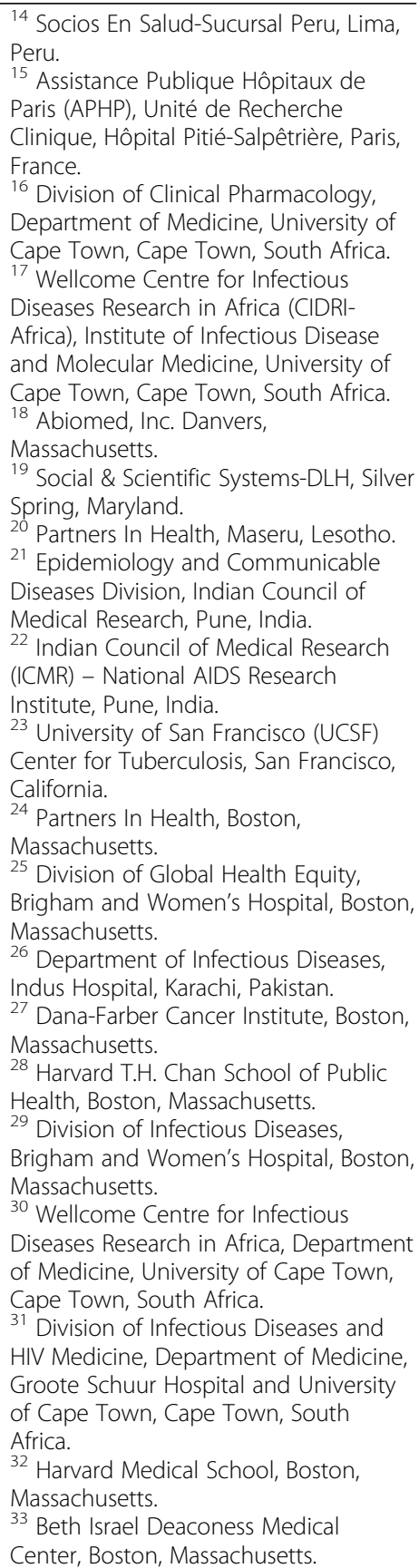 \\
\hline
\end{tabular}

Name and contact information for the trial sponsor $\{5 \mathrm{~b}\}$

Role of sponsor $\{5 c\}$

\section{Administrative information (Continued)}

\begin{tabular}{|c|c|}
\hline Title $\{1\}$ & $\begin{array}{l}\text { Evaluating newly approved drugs } \\
\text { for multidrug-resistant tuberculosis } \\
\text { (endTB): study protocol for an adap- } \\
\text { tive, multi-country randomized con- } \\
\text { trolled trial }\end{array}$ \\
\hline & $\begin{array}{l}\text { writing/submission of the report. The } \\
\text { sponsor has ultimate authority over } \\
\text { these activities. The sponsor is } \\
\text { responsible for (delegation of) } \\
\text { communicating protocol modifications } \\
\text { to investigators, participants, and } \\
\text { registries after regulatory and ethics } \\
\text { approvals. The design and } \\
\text { implementation of the trial are } \\
\text { supported and overseen by the } \\
\text { following external, independent } \\
\text { committees: a Data Safety Monitoring } \\
\text { Board, a Scientific Advisory Committee, } \\
\text { the Global Tuberculosis Community } \\
\text { Advisory Board (TB-CAB). }\end{array}$ \\
\hline
\end{tabular}

\section{Background \{6a\}}

The endTB trial (evaluating newly approved drugs for multidrug-resistant tuberculosis, clinicaltrials.gov Identifier NCT02754765) was designed in 2015 to avail of and optimize exciting, new developments in treatment for drug-resistant tuberculosis (DR-TB). That year, there were an estimated 580,000 new cases of TB caused by organisms resistant to the most potent first-line anti-TB drugs (at least rifampin, RR-TB; or at least isoniazid and rifampin, MDR-TB). Only 132,120 cases (22.7\%) of MDR/RR-TB were detected and 125,000 (21.5\%) received treatment with second-line TB drugs. Roughly half were cured [1]. The consequences of this situation are dire: death is common among MDR/RR-TB patients who experience unfavourable treatment outcomes [2, 3]. Many of those treated receive conventional regimens comprising 18-24 months of toxic, poorly tolerated multidrug treatment [4]. Cure is reported in only $57 \%$ globally and loss to follow-up occurs in $16 \%[5,6]$. Toxicity occurs in nearly all patients receiving conventional treatment for MDR-TB [7]. In two pivotal trials of new anti-TB drugs, patients in the control arms received placebo plus background conventional MDR-TB treatment regimens: approximately 95\% experienced adverse events. And serious adverse events were reported in 19\% of participants randomized to the control arm in the bedaquiline trial (NCT00449644) and 9\% in the delamanid trial (NCT00685360) [8, 9]. Observational cohorts corroborate these findings, with 73 to $79 \%$ reported to experience toxicity $[10,11]$. This complicates the management of MDR-TB treatment and frequently leads to suspension and replacement of drug(s) in conventional regimens $[12,13]$. Toxicity is a major driver of loss to follow-up [14] and is associated with lower rates of culture conversion [15]. 
Recent developments offered promise of improvement; however, this potential has yet to be fully realized. A shorter alternative regimen with promising efficacy emerged for MDR-TB in patients with limited prior treatment exposure and resistance [16, 17]. The "STREAM" or "Bangladesh" regimen relies on drugs commonly used in first-line treatment and/or in MDR/ RR-TB treatment for 20 years; in some settings, resistance to these drugs is common [18, 19]. Uptake of this innovation was limited by this reality and also by the simultaneous emergence of the first new anti-TB drugs in 50 years. In 2012 and 2014, bedaquiline and delamanid, respectively, received conditional approval from stringent regulatory authorities (SRA) for the treatment of MDR-TB. The pivotal trials of these drugs added each to a background regimen, improving interim and longterm outcomes, but retaining the toxic, long, conventional (injectable-containing) regimen $[8,9,20,21]$. Murine and clinical studies supported the shortening potential of combinations of new agents, coadministered with repurposed drugs [22-31]. Such combinations, however, have not been optimized and examined rigorously through randomized, internally, concurrently controlled clinical trials with participants followed to ascertain relapse-free cure. Nevertheless, there has been substantial pressure to modify guidance in this breach between the unpalatable conventional regimen and rigorously studied shorter, injectablesparing regimens. Policy changes have, therefore, generally comprised "conditional recommendations" based on "very low-quality" evidence. The endTB trial aims to close this gap by studying in a randomized, internally controlled trial, shorter treatments composed of novel combinations of newer and repurposed drugs. The endTB trial tests experimental regimen combinations drawn from among the following drugs: bedaquiline, delamanid, clofazimine, linezolid, moxifloxacin or levofloxacin, and pyrazinamide. endTB regimen selection was guided by the following principles. Regimens should: contain at least one new drug class, include 3 to 5 likely effective drugs preferentially those without extensive prior use, be effective against a range of MDR/RR strains of M. tuberculosis, shorten duration, be all-oral, and have a simple dosing profile, acceptable side effect profile, and minimal drug-drug interactions with antiretrovirals [32]. This phase 3 trial evaluates five novel, 9month, all oral, treatment regimens for patients with fluoroquinolone-susceptible MDR/RR-TB. The study protocol is presented here.

\section{Objectives $\{7\}$}

The primary objective of the endTB trial is to assess whether the efficacy of experimental regimens at 73 weeks post-randomization is non-inferior to that of the control. Secondary objectives include efficacy comparisons at 8,39 , and 104 weeks. Safety objectives include comparisons between the experimental and control arms of the proportion of patients who died or who experience grade 3 or higher adverse events (AEs), serious adverse events (SAEs) of any grade, and adverse events of special interest (AESI), at 73 and 104 weeks. Important exploratory objectives include comparing the effect of two linezolid dose-reduction strategies on toxicity and efficacy of experimental regimens.

\section{Methods}

The study protocol hereby presented contains all items defined by the Standard Protocol Items: Recommendations for Interventional Trials (SPIRIT) statement. A completed SPIRIT checklist is provided (Supplement 1).

\section{Design $\{8\}$}

endTB is a phase III, pragmatic, multi-country, adaptive, randomized, controlled, parallel, non-inferiority openlabel clinical trial evaluating the efficacy and safety of five 9-month treatment regimens containing recently approved drugs for MDR-TB compared to a control that is the current standard of care for MDR-TB.

\section{Randomization, masking, and blinding $\{16 a\}\{16 b\}\{16 c\}\{17 a\}$} Randomization is web-based (with voice backup), and adapted randomization lists are uploaded to the webbased platform. The study is open label; the regimens are not masked. Microbiology staff who perform testing that is core to efficacy assessments are blinded to treatment assignment. Central investigators are also blinded. Since site investigators are not blinded to assignment and are possibly influenced by opinions about regimen allocation, permanent regimen changes are made with input from the independent Clinical Advisory Committee (CAC), staffed by expert MDR-TB clinicians. The $\mathrm{CAC}$ also validates study endpoints that are assigned by local investigators. CAC members do not provide any input on the study protocol and are not involved in the study analysis.

Secondary, balanced (1:1 allocation ratio) randomization to linezolid dose-reduction strategy occurs among patients in the experimental arms at 16 weeks post randomization or earlier if required for toxicity.

\section{Interim analysis and stopping \{21b\}}

Randomization is outcome adaptive using interim Bayesian analyses of efficacy endpoints; it has been described previously $[33,34]$ and is summarized here. The initial randomization list used fixed block sizes with balanced allocation to all 6 arms. After approximately 30 
participants are assigned to each arm, the response adaptation begins. At approximately monthly intervals, interim treatment effect (at 8 and 39 weeks) is estimated for each arm, relative to the control, through Bayesian modelling. Randomization probabilities are updated after each interim analysis; higher probability of randomization is assigned to arms with greater interim treatment effect. The probability of assignment to the control arm matches the probability of assignment to the most effective experimental arm. The interim analysis is conducted by a Bayesian statistician (MC) who is not involved in study operations.

Further efficacy review may inform dropping of regimen(s) for futility. Stopping an arm early may be indicated if the posterior probability of a regimen being non-inferior to the control at week 73 falls below a predefined threshold of $5 \%$. Conversely, if the posterior probability is at least $95 \%$ that a regimen is superior to the control at week 73, stopping for efficacy will be considered. This may trigger a review by the DSMB to decide if this superior regimen should be stopped (i.e. no more participants are randomized to this regimen) or if the evidence is to be balanced with the safety data.

\section{Regimen composition and duration experimental arms $\{11 a\}$}

Experimental arms comprise standardized, four- or fivedrug combinations of the following: delamanid, clofazimine, linezolid, pyrazinamide, and a fluoroquinolone (moxifloxacin or levofloxacin); see Tables 1 and 2 for composition and dosing, respectively. Selected regimens combine drugs with distinct mechanisms of action, bactericidal (bedaquiline, delamanid, linezolid, fluoroquinolones) and sterilizing (bedaquiline, clofazimine, delamanid, linezolid, pyrazinamide [35-37]) activity against Mycobacterium tuberculosis, and activity in different microenvironments (acid, hypoxic, etc.). Possible overlapping toxicity, particularly QT interval prolongation that could predispose to cardiotoxicity, is managed by including no more than 2 drugs considered to be major QT interval prolongers (bedaquiline, clofazimine, and moxifloxacin) in a regimen. All regimens contain a fluoroquinolone and pyrazinamide, the only drugs to which prior exposure is widespread. The fluoroquinolones, especially the later-generation class members, are a cornerstone of treatment for MDR-TB. In vitro, animal, and human studies all support the inclusion of this drug class for the treatment of resistant TB $[38,39]$ in patients with isolates susceptible to fluoroquinolones. Although pyrazinamide is linked to toxicity, especially hepatotoxicity, WHO previously recommended inclusion of pyrazinamide in all MDR-TB regimens [40]. Currently, it is recommended to count pyrazinamide as an effective drug only in case drug susceptibility testing confirms susceptibility [4]. Acknowledging the important role of pyrazinamide (when active) in TB treatment shortening [41], and the uncertainty about activity at the time of treatment initiation, all endTB experimental regimens contain pyrazinamide plus at least three other likely effective anti-TB agents. This is consistent with WHO guidance on the treatment of MDR-TB in place when the trial was designed [42].

Treatment in experimental arms is for 39 weeks; participants in the experimental arms are allowed up to 47 weeks to complete the 39-week treatment course. Of note, while linezolid dosing starts at $600 \mathrm{mg} /$ day, the aforementioned dose-reduction randomization assigns experimental arm patients to receive either linezolid at $300 \mathrm{mg}$ daily or at $600 \mathrm{mg}$ three times/week starting at 16 weeks (or earlier, if indicated by toxicity).

\section{Regimen composition and duration control arm $\{6 b\}$}

Control-arm treatment is constructed according to the latest WHO recommendations [4] and local guidance: composition of the regimens may therefore change over the course of the trial. Control-arm treatment may contain one or both recently approved drugs (bedaquiline or delamanid) in addition to companion drugs. Duration is variable: the conventional regimen is delivered for approximately 86 weeks. Shorter regimens that are endorsed by WHO for routine use, are permitted [43]. Oral drugs are delivered 7 days/week in both experimental and control arms. Injectable drugs (used only rarely under protocol version 3.3) in the control arm are delivered at least 6 days/week, according to local practices. All drug intakes are directly observed.

Table 1 Description of endTB treatment arms $\{11 \mathrm{a}\}$

\begin{tabular}{|c|c|c|c|c|c|c|}
\hline Trial regimens & Bedaquiline & Delamanid & Clofazimine & Linezolid & Fluoroquinolone & Pyrazinamide \\
\hline endTB 1 & $\mathrm{Bdq}$ & & & Lzd & Mfx & Z \\
\hline endTB 2 & Bdq & & $\mathrm{Cfz}$ & Lzd & Lfx & Z \\
\hline endTB 3 & Bdq & $\mathrm{D} / \mathrm{m}$ & & Lzd & Lfx & Z \\
\hline endTB 4 & & $\mathrm{Dlm}$ & $\mathrm{Cfz}_{\mathrm{Z}}$ & Lzd & Lxf & Z \\
\hline endTB 5 & & $\mathrm{D} / \mathrm{m}$ & $\mathrm{Cfz}$ & & Mfx & Z \\
\hline endTB 6 (Control) & \multicolumn{6}{|c|}{ Standard of care control, composed according to latest WHO Guidelines, including the possible use of DIm or Bdq. } \\
\hline
\end{tabular}


Table 2 Experimental arm drug doses by weight bands $\{11 \mathrm{a}\}$

\begin{tabular}{|c|c|c|c|c|c|}
\hline \multirow[t]{2}{*}{ Drug $^{a}$} & \multicolumn{5}{|c|}{ Weight band (kg) } \\
\hline & $30-35$ & $>35-45$ & $>45-55$ & $>55-70$ & $>70$ \\
\hline Bedaquiline & \multicolumn{5}{|c|}{400 mg QD x 2 weeks $^{\text {b }}$ followed by 200 mg 3x/week } \\
\hline Delamanid & \multicolumn{5}{|c|}{100 mg BID } \\
\hline Moxifloxacin & \multicolumn{5}{|l|}{$400 \mathrm{mg}$} \\
\hline Levofloxacin & $750 \mathrm{mg}$ & $1000 \mathrm{mg}$ & & & \\
\hline Linezolid $^{c}$ & \multicolumn{5}{|c|}{600 mg QD up to week 16 (followed by 300 mg QD or 600 mg 3x/week) } \\
\hline Clofazimine & \multicolumn{5}{|l|}{$100 \mathrm{mg}$} \\
\hline Pyrazinamide & $800 \mathrm{mg}$ & $1200 \mathrm{mg}$ & $1600 \mathrm{mg}$ & & $2000 \mathrm{mg}$ \\
\hline
\end{tabular}

${ }^{\mathrm{a}}$ Dosing is once a day unless otherwise indicated

${ }^{\mathrm{b}}$ The loading dose of bedaquiline treatment comprises 2 weeks of $400 \mathrm{mg} \mathrm{QD}$. If the patient is already receiving bedaquiline treatment, bedaquiline within the experimental regimen should be dosed as a continuation of that treatment (i.e. only remaining doses of the 2-week loading dose should be administered and a load should not be restarted if it was already completed at time of study treatment initiation)

${ }^{c}$ Linezolid dosing is routinely modified at week 16 or sooner if necessary to reduce toxicity related to linezolid. The modification entails either decreased ( 300 mg daily) or intermittent ( $600 \mathrm{mg} 3 \times /$ week) dosing as defined by balanced randomization

\section{Follow-up duration}

Study participation is for up to 104 weeks postrandomization; those participants remaining in followup when the last participant completes 73 weeks will have their follow-up truncated.

\section{Setting $\{9\}$}

The endTB trial is jointly coordinated by members of the endTB consortium: Interactive Research and Development (IRD), Médecins Sans Frontières (MSF), and Partners In Health (PIH) and their research partners, Harvard Medical School, Epicentre, and the Institute of Tropical Medicine of Antwerp (ITM); most coordination is based at MSF. The trial is implemented in countries selected for the following: a significant burden of MDR/RR-TB; the presence of a member institution of the endTB consortium or another entity experienced in TB clinical trials and an existing relationship between TB services and the aforementioned group; clinical trial experience or potential (established through a multi-step siteassessment process); suitable MDR-TB clinical management systems, regulatory environment, research pharmacy capability, and micro/molecular biology services; and heterogeneity in DR-TB patient characteristics (geography, resistance, comorbidities, risk-factor profiles). The list of participating countries and sites is available on ClinicalTrials.gov.

\section{Study population}

\section{Inclusion/exclusion $\{10\}$}

The study population comprises males and females aged $\geq 15$ years with pulmonary TB suspected or confirmed to be resistant to rifampin, without known fluoroquinolone resistance. Inclusion and exclusion criteria are detailed in Table 3. Inclusion criteria include pulmonary TB caused by fluoroquinolone-susceptible and rifampin- resistant $M$. tuberculosis, written informed consent, willingness to use effective contraception, and expected ability to remain locatable in study catchment area for the duration of study participation. Patients are excluded for known allergies or hypersensitivity to any of the investigational drugs; pregnancy or breastfeeding; prior

Table 3 Inclusion and exclusion criteria for study.

\section{Inclusion criteria}

- Documented pulmonary TB due to strains of Mycobacterium tuberculosis resistant to rifampin and susceptible to fluoroquinolones

- Diagnosed by validated rapid molecular test

$\cdot \geq 15$ years of age

- Willingness to use effective contraception

- Provision of informed consent for study participation

- Residence in a dwelling that can be located by study staff and an expectation to remain in the area for the duration of the study

\section{Exclusion criteria}

- Patients with known allergies or hypersensitivity to any of the investigational drugs

- Patients known to be pregnant or unwilling or unable to stop breastfeeding an infant

- Patients unable to comply with treatment or follow-up schedule - Patients with exposure (intake for 30 days or more) in the past 5 years to bedaquiline, delamanid, linezolid, or clofazimine, or with proven or likely resistance to bedaquiline, delamanid, linezolid, or clofazimine - Patients who have received second-line drugs for 15 days or more prior to the screening visit date in the current MDR-TB treatment episode

- Patients with one or more of the following laboratory results:

- Grade 3 or higher haemoglobin, calcium, magnesium, creatinine, or bilirubin

- Grade 2 or higher potassium, aspartate aminotransferase, alanine aminotransferase, or total bilirubin

- Albumin $<2.8 \mathrm{~g} / \mathrm{dL}$

- Grade 4 result on any other screening laboratory tests

- Patients with cardiac risk factors including ECG abnormalities (i.e. $\mathrm{QTCF} \geq 450 \mathrm{~ms}$ ), pacemaker implant, and personal/family history of cardiovascular disease (i.e. long QT syndrome, left or right bundle branch block)

- Patients requiring continued use of a contraindicated medication

- Patients currently taking part in another trial of medicinal product

- Patients with any condition (social or medical) which, in the opinion of the investigator, would make study participation unsafe 
exposure/resistance to bedaquiline, delamanid, linezolid, or clofazimine; any second-line anti-TB drug exposure for 15 days or more immediately prior to the screening visit date; abnormal haematology, biochemistry; cardiac risk factors; required use of a contraindicated medication; social or medical conditions, which, in the opinion of the investigator, would make study participation unsafe. HIV positivity, regardless of CD4 lymphocyte counts, is not a reason for exclusion. Fluoroquinolone resistance on phenotypic test will result in late exclusion.

\section{Study treatment discontinuation and study withdrawal $\{11 b\}\{11 d\}$}

Study treatment may be discontinued in the following situations: (1) pregnancy or breastfeeding, (2) required use of prohibited concomitant medications, (3) indications of treatment failure, and (4) any other condition (social or medical) which the site principal investigator believes would make study participation unsafe. Prohibited concomitant medications depend on treatment received by the participant. They include moderate and strong CYP3A4 inhibitors and inducers for bedaquilinecontaining regimens; strong inducers are also disallowed with delamanid-containing regimens. With linezolidcontaining regimens, disallowed medications are any medicinal product that inhibits monoamine oxidases A or B, tricyclic antidepressants, selective serotonin reuptake inhibitors, selective serotonin/norepinephrine reuptake inhibitor, triptans, and other serotoninergic agents. Decisions to permanently discontinue study treatment are made in consultation with the Clinical Advisory Committee. Participants will be referred to local services for treatment. Discontinuation of treatment for the abovespecified reasons, and alteration of the assigned study regimen, results in withdrawal from study. Study participation also ends if consent is withdrawn. In case study participation is ended prematurely, an early termination visit will be performed if possible. In addition, participants discontinuing treatment before the week 73 visit will be encouraged to perform post-termination follow-up visits at weeks 39 and 73, as needed.

\section{Recruitment and retention $\{11 c\}\{15\}\{18 b\}\{26 a\}\{22\}\{30\}$}

Prospective participants are identified by facility staff in inpatient or outpatient facilities, located in the study catchment areas, that provide $\mathrm{TB}$ diagnosis and/or treatment. Patients who agree to be evaluated for the study are referred to study staff. Study staff explain the study, including potential risks and benefits associated with participation. Subsequently, screening consent is obtained from participants (or from parent or guardian, in case of minors, who also provide assent) by site investigators or other delegated site staff prior to any trial-specific evaluation. Baseline consent and randomization follow in those who are eligible.

Retention in the study is ensured through comprehensive, individualized patient support, including adherence enablers and home visits as needed. During treatment, adherence is monitored at every visit and adherence counselling is provided by specialized staff. All transport costs encountered by participants are covered by the study. Food support is provided. Participants requiring care for comorbidities (e.g. HIV, diabetes mellitus) receive all care in the study setting or through facilitated referrals to local providers.

Adverse events are solicited at all study visits; spontaneous reporting of adverse events can also occur at scheduled study visits, through daily treatment support, or at unscheduled visits. Adverse events are managed according to grade and relatedness to study drug; closer monitoring may be recommended at any grade. Investigators are encouraged to modify or withhold study drugs possibly related to adverse events of grade 3 or higher. Additional guidance is provided in study standard operating procedures and by the CAC.

\section{Study endpoints $\{12\}$ \\ Efficacy}

The primary efficacy outcome is the proportion of participants with favourable outcome at week 73 (Table 4).

Secondary efficacy outcomes are:

1. The proportion of participants with favourable outcome at week 39

2. The proportion of participants with favourable outcome at week 104

3. The proportion of patients who experienced failure or relapse at week 73 and at week 104

4. Early treatment response, which is assessed through the following: (a) proportion of patients with culture conversion at 8 weeks assessed in Mycobacteria Growth Indicator Tube (MGIT) culture method (and on Löwenstein-Jensen [LJ] culture medium where possible); (b) time to culture conversion assessed in MGIT system (and LJ where possible); and (c) change in time to positivity (TTP) in MGIT over 8 weeks

The definitions used for the primary efficacy outcome are shown in Table 4. Efficacy endpoints at weeks 39, 73 , and 104 are validated by the CAC, the aforementioned committee of expert MDR-TB clinicians who do not provide any input on the study protocol and are not involved in the study analysis.

Although differences are not expected, the primary efficacy endpoints are also used to evaluate efficacy across linezolid-dose-reduction strategies. 
Table 4 Definition of primary treatment outcome $\{12\}$

\begin{tabular}{|c|c|c|}
\hline Outcome definition & Definition of favourable outcome & Definition of unfavourable outcome \\
\hline $\begin{array}{l}\text { Proportion of participants } \\
\text { with a favourable } \\
\text { outcome at week } 73\end{array}$ & $\begin{array}{l}\text { The outcome is not classified as unfavourable, and one of } \\
\text { the following is true: } \\
\text { 1. The last two culture results are negative. These two } \\
\text { cultures must be taken from sputum samples collected on } \\
\text { separate visits, the latest between weeks } 65 \text { and } 73 \text {. } \\
\text { 2. The last culture result (from a sputum sample collected } \\
\text { between weeks } 65 \text { and } 73 \text { ) is negative, and either there is } \\
\text { no other post-baseline culture result or the penultimate cul- } \\
\text { ture result is positive due to laboratory cross contamination, } \\
\text { and bacteriological, radiological and clinical evolution is } \\
\text { favourable. } \\
\text { 3. There is no culture result from a sputum sample } \\
\text { collected between weeks } 65 \text { and } 73 \text { or the result of that } \\
\text { culture is positive due to laboratory cross contamination, } \\
\text { and the most recent culture result is negative, and } \\
\text { bacteriological, radiological, and clinical evolution is } \\
\text { favourable. }\end{array}$ & $\begin{array}{l}\text { Any of the following: } \\
\text { 1. Replacement or addition of one or more investigational } \\
\text { drugs in an experimental arm (failure). } \\
\text { 2. Replacement or addition of two or more investigational } \\
\text { drugs in the control arm (failure). } \\
\text { 3. Initiation of a new MDR-TB treatment regimen after the } \\
\text { end of the allocated study regimen and before week } 73 \\
\text { (relapse). } \\
\text { 4. Death from any cause. } \\
\text { 5. At least one of the last two cultures, the latest being } \\
\text { from a sputum sample collected between weeks } 65 \text { and } 73 \text {, } \\
\text { is positive in the absence of evidence of laboratory cross } \\
\text { contamination (failure/relapse). } \\
\text { 6. The last culture result (from a sputum sample collected } \\
\text { between weeks } 65 \text { and } 73 \text { ) is negative; AND there is no } \\
\text { other post-baseline culture result or the penultimate culture } \\
\text { is positive due to laboratory cross contamination; and bac- } \\
\text { teriological, radiological, or clinical evolution is unfavourable } \\
\text { (failure/relapse). } \\
\text { 7. There is no culture result from a sputum sample } \\
\text { collected between weeks } 65 \text { and } 73 \text { or it is positive due to } \\
\text { laboratory cross contamination. } \\
\text { AND the most recent culture is negative; and } \\
\text { bacteriological, radiological or clinical evolution is } \\
\text { unfavourable (failure/relapse); or the most recent culture } \\
\text { result is positive in the absence of laboratory cross } \\
\text { contamination. } \\
\text { 8. The outcome is not assessable because there is no } \\
\text { culture result from a sputum sample collected between } \\
\text { weeks } 65 \text { and } 73 \text { or it is positive due to laboratory cross } \\
\text { contamination. } \\
\text { AND } \\
\text { - There is no other post-baseline culture result or the most } \\
\text { recent culture is positive due to laboratory cross } \\
\text { contamination. } \\
\text { - The most recent culture is negative and bacteriological, } \\
\text { radiological, and clinical evolution is not assessable. } \\
\text { 9. Previously classified as unfavourable in the present study } \\
\text { (except for participants whose outcome at } 39 \text { weeks was } \\
\text { unfavourable because it was unassessable). }\end{array}$ \\
\hline
\end{tabular}

\section{Safety}

The main safety outcomes are at 73 (and 104) weeks:

1. The proportion of patients who died of any cause

2. The proportion of participants with grade 3 or greater AEs, SAEs, and AESIs of any grade by 73 and 104 weeks (grading is determined using the MSF Severity Scale, which was derived from CTCAE v4.0, supplemented by DMID and DAIDS scales where necessary [44]. The following adverse events, at grade 3 or higher, are defined as AESI: "electrocardiogram QT corrected interval prolonged"; leukopenia, anaemia or thrombocytopenia; peripheral neuropathy; optic neuritis; and increase in alanine aminotransferase (ALT) or aspartate aminotransferase (AST).)

The endpoint for assessment of safety of the linezoliddose-reduction strategies is severe linezolid-related toxicity, defined as grade 3 or higher linezolid-related AEs (leukopenia, anaemia, thrombocytopenia, peripheral neuropathy, and optic neuropathy), SAEs, and AEs requiring linezolid discontinuation.

\section{Analysis of the primary endpoint and analysis populations $\{20 a\}\{20 b\}\{20 c\}$}

The primary analysis will compare the proportions of participants with a favourable outcome at week 73 between each experimental arm and the control arm: for all pairwise comparisons, a two-sided 95\% confidence interval of the difference will be estimated. The noninferiority of an experimental arm compared to the control will be established if the difference in favourable outcome at week 73 is greater than the lower equivalence margin, i.e. if the lower bound of the one-sided 97.5\% CI-which corresponds to the lower bound of the 2 -sided $95 \% \mathrm{CI}$-is greater than or equal to $-12 \%$. We present two main reasons for the $12 \%$ non-inferiority margin. First, the $70 \%$ expected efficacy of the control regimen was already higher than that of the standard of 
care regimen, mitigating concerns about biocreep. Emerging data has suggested that the control arm, containing new and repurposed drugs, may be even more effective (e.g. $80 \%)[16,45]$. If so, any regimen established as noninferior could be concluded, with $97.5 \%$ confidence, to have a favourable outcome frequency of at least $68 \%$. Second, relative to the control arm and conventional regimens, the experimental regimens would result in a significantly reduced pill burden and treatment duration, expected improved tolerability, and increased adherence.

The family-wise type I error will be controlled by ordering the non-inferiority comparisons. A fixed sequence approach will be considered: the regimen with the highest proportion of favourable outcomes will be compared to the control. If non-inferiority is concluded, then a comparison between the control and the experimental regimen having the second highest proportion of favourable outcomes will be performed, and so on as long as non-inferiority is concluded. Once noninferiority is not demonstrated, the comparisons will stop. All the previous comparisons will have been done at the full one-sided alpha level of $2.5 \%$. Adjusted analyses on the primary endpoint will be also performed by controlling for covariates.

The main primary efficacy analyses will be performed on both modified intent-to-treat (mITT) and perprotocol (PP) populations for the non-inferiority comparisons. The mITT population contains all randomized participants with culture-positive, FQ-susceptible, and RR-TB. The PP population contains participants from the mITT population who completed the study without any major protocol deviation that impacts the assessment of the primary endpoint. Patients who discontinue the study for reasons other than death or loss to followup will be excluded from the PP. Sensitivity analyses will be performed in two other efficacy populations: the assessable population and the mITT plus those with baseline culture-negative tuberculosis.

Safety analyses will be performed on the safety population, which includes all enrolled participants who had received at least one dose of study treatment (as treated). A full description of the statistical methods, including handling of missing data and planned analyses, is detailed in the Statistical Analysis Plan.

\section{Sample size assumptions $\{14\}$}

The sample size was estimated through simulations that considered (1) early efficacy responses (at weeks 8 and 39), (2) primary outcome (at week 73) with variations described below, (3) the expected number of noninferior experimental arms, (4) the type I error $(2.5 \%$ one-sided), (5) expected reductions in population from ITT to mITT (11\%) and mITT to PP (10\%), and (6) the non-inferiority margin (12\%). Sample size estimates were calculated for $75 \%$ favourable outcome at 73 weeks in the non-inferior experimental arms. Favourable outcome frequency in the control arm was assumed to be $70 \%$ at 73 weeks. A sample size of 750 randomized participants provides power greater than $80 \%$ to demonstrate the non-inferiority of at least 1 (and up to 3) experimental regimens. The expected 75\% 73-week response in the experimental arm reflects available data. This includes conventional-regimen treatment with delamanid that resulted in $74.5 \%$ favourable treatment outcome [20] and observational studies of short-course MDR-TB treatment that reported treatment success of greater than $80 \%$ [46-49]. The sample size simulations were performed in R (R Foundation for Statistical Computing, Vienna, Austria) using bootstrapping methods.

\section{Data collection, monitoring, and management $\{18 a\}\{19\}\{21$ a $\}\{22\}\{23\}\{27\}$}

Data are collected and entered into an electronic case report form, in a web-based system that is compliant with International Conference on Harmonisation (ICH) Good Clinical Practice (GCP) guidelines. Range checks are programmed into the system. Designated study team members at each participating site perform real-time quality control and periodic quality assurance activities. Checks for consistency are implemented at the data entry level on site and centrally after data entry. Regular data review and data cleaning for quality control are organized in a blinded way and detailed in the Data Monitoring Plan. In addition, external monitoring is performed in accordance with the protocol specific requirements, ICH GCP guidance, and other applicable requirements.

Data are managed centrally by Epicentre. Additionally, safety data are also entered in a separate pharmacovigilance database at the centralized MSF Pharmacovigilance Unit. Appropriate medical and research records are maintained for the trial, in compliance with ICH E6 GCP and regulatory and institutional requirements. All study documents are coded with a study identification number. All study records are managed in a secure and confidential fashion.

All adverse events that occur during study are documented and followed to resolution or stabilization; in the case of AESIs and SAEs, this follow-up may extend beyond the normal study-reporting period. SAEs are notified, within $24 \mathrm{~h}$ of awareness, by the site principal investigator (or designee) to the MSF Pharmacovigilance Unit. All SAEs deemed related to one or more investigational product(s) and considered unexpected with the use of such products are reported to National Regulatory Authorities and national/local IRBs. All other SAEs are reported in an Annual Safety Report prepared 
by the MSF Pharmacovigilance Unit, and earlier if there are specific local regulations for more frequent reporting. In addition, safety data are reviewed semi-annually by an independent Data Safety Monitoring Board (DSMB), the members of which have expertise in clinical trials, MDR-TB, pharmacology, Bayesian adaptive randomization, and electrophysiology. Their functioning is detailed in the DSMB charter.

\section{Participant timeline}

Figure 1 shows the schedule of eligibility assessment, visit procedures, and assessments over up to 104 weeks of study participation.

\section{Dissemination of trial findings $\{29\}\{31 \mathrm{a}\}\{31 \mathrm{~b}\}$}

The results of the trial will be disseminated under the responsibility of the principal investigators of the study. Investigators/study authors will have full access to the final trial dataset. Trial results will be published in peerreviewed scientific journals and presented at national and international conferences, as appropriate. Results will be shared and discussed with study participants and affected communities. Authorship will be defined according to International Committee of Medical Journal Editors criteria. No professional writers will be involved.

\section{Biological specimens $\{26 \mathrm{~b}\}\{33\}$}

Subjects (and their legal representatives as applicable) are asked to provide written informed consent for storage and future use of M. tuberculosis isolates obtained from sputum samples. A subject may consent to study participation without consenting to future use of stored isolates. Stored isolates may be used only to improve diagnosis (including resistance testing) and treatment of TB. Relevant IRBs will oversee any future research using these specimens.

\section{Discussion}

The lack of safe, effective, short, simple regimens for treatment of RR-/MDR-TB is a major challenge in $\mathrm{TB}$ control. Research shows that inadequately treated drugresistant TB can undermine the population-level benefits of high rates of success in treatment of drug-susceptible TB [50]. Moreover, conventional, long, injectablecontaining regimens have significant, negative quality-oflife impacts on patients with MDR-TB [51]. Shorter regimens have the potential to reduce loss to follow-up rates and treatment cost and can increase treatment access [52]. To maximize the potential impact of such shorter regimens on the existing gaps, the endTB trial uses an innovative approach. Instead of trying to select one single regimen-or an algorithm to develop individualized regimens-for all MDR-TB cases, endTB aims to identify multiple new regimens against FQ-susceptible TB as rapidly as possible. The trial assesses non-inferior (rather than superior) efficacy of the experimental regimens because of the significant benefits (detailed below) that could accrue to patients and health systems even in the absence of improved efficacy.

If safe and effective, endTB's novel regimens have the potential to improve the current treatment options significantly. In the many settings that have not yet

\begin{tabular}{|c|c|c|c|c|c|c|c|c|}
\hline \multirow[b]{3}{*}{ TIMEPOINT } & \multicolumn{8}{|c|}{ STUDY PERIOD } \\
\hline & \multirow{2}{*}{$\begin{array}{c}\text { Screening } \\
\text { Max } 14 \text { days } \\
\text { before } \\
\text { Randomization }\end{array}$} & \multirow{2}{*}{$\begin{array}{c}\begin{array}{c}\text { Baseline and } \\
\text { Randomization }\end{array} \\
\text { to }\end{array}$} & \multicolumn{5}{|c|}{ Post-allocation } & \multirow{2}{*}{$\begin{array}{c}\text { Close-ou } \\
\text { W104 }\end{array}$} \\
\hline & & & w1 & w8 & w39 & w47 & $w 73$ & \\
\hline \multicolumn{9}{|l|}{ ENROLMENT: } \\
\hline \multirow{3}{*}{$\begin{array}{l}\text { Eligibility assessment } \\
\text { Informed consent } \\
\text { Treatment Allocation }\end{array}$} & $\mathrm{x}$ & $\mathrm{x}$ & & & & & & \\
\hline & $x$ & $\mathrm{x}$ & & & & & & \\
\hline & & $\mathrm{x}$ & & & & & & \\
\hline \multicolumn{9}{|l|}{ INTERVENTIONS: } \\
\hline Clinical Evaluations & $x$ & $x$ & & & & & & \\
\hline \multirow[t]{2}{*}{ Specialist Examinations } & & $x$ & & & & & & \\
\hline & $x$ & $x$ & & & & & & \\
\hline \multirow{2}{*}{$\begin{array}{l}\text { Sputum Specimen } \\
\text { Testing } \\
\text { Blood and Urine Testing }\end{array}$} & $x$ & $\mathrm{X}^{*}$ & & & & & & \\
\hline & $\mathrm{x}$ & $x$ & & $\leftarrow$ & & & & \\
\hline \multirow{3}{*}{$\begin{array}{l}\text { ASSESSMENTS: } \\
\text { [Primary outcome } \\
\text { assessment] } \\
\text { [Secondary outcome } \\
\text { assessment] }\end{array}$} & & & & & & & & \\
\hline & & & & & & & $x$ & \\
\hline & & & & $x$ & $x$ & & & $x$ \\
\hline
\end{tabular}

Fig. 1 Schedule of enrolment, interventions, and assessments $\{12\}\{13\}$. *Sputum tests can be repeated at the baseline visit if needed for eligibility assessment 
adopted all-oral, shorter regimens, endTB regimens could reduce treatment duration by more than $50 \%$, eliminate injections and lessen the pill burden, and potentially increase efficacy without worsening toxicity. Some countries, including South Africa, have adopted routine use of shorter regimens in which bedaquiline has replaced the daily injectable $[4,53,54]$. This was based on encouraging results from observational research. To date, this evidence has been graded as low or very low certainty and WHO recommendations have been conditional for use of such regimens [4]. Relevant for settings that have hesitated, as well as for those that have adopted these innovations, the endTB trial will provide high-quality, direct evidence about specific regimens compared to the current standard of care.

The endTB trial complements other efforts, recently completed, planned or ongoing, to optimize treatment for drug-resistant TB. In addition, a pharmacokinetic substudy will inform optimized dosing and offer insights into pharmacokinetic and pharmacodynamic drug-drug interactions. endTB focuses on questions, populations, or regimens and/or provides additional (high-quality) evidence compared to other studies. For example, OptiQ (NCT01918397) optimized the dose of levofloxacin [55]; when available, efficacy findings from that study could be incorporated into use of any levofloxacincontaining endTB regimens found to be non-inferior to the current standard of care. The endTB observational study expanded the use of novel compounds (bedaquiline and delamanid) and repurposed older agents (linezolid and clofazimine) that had limited prior population exposure for TB. Delivered within longer and sometimes injectable-sparing regimens, preliminary results are encouraging [56]. Shorter regimens are the subject of other studies. STREAM 1 established the non-inferiority of the shorter "Bangladesh" regimen to an internal control that did not include treatment innovations that were occurring concurrently with the trial (e.g. use of bedaquiline, delamanid, or linezolid). And, toxicity of the STREAM regimen was found to be significant [16]. STREAM II (NCT02409290) adds bedaquiline to regimens (longer and shorter) containing drugs that have been used historically for DR-TB.

A more recent development, the SRA licensure of pretomanid was the first marketing authorization for a novel agent (in the same nitroimidazole class as delamanid) used within a new, shorter regimen. Prospects are promising for this regimen containing pretomanid, bedaquiline, and linezolid (BPaL); currently WHO recommends its use only under research conditions [4]. Additional research is required in light of certain characteristics of the pivotal study: the small sample size $(N=109)$; lack of an internal, concurrent control; single-country setting; and the high rate of toxicity with the dose of linezolid examined [57-60]. The uncontrolled ZeNix trial, expected to report in 2021, aims to optimize linezolid dosing within the BPaL regimen (NCT03086486) in a more heterogeneous patient population. Lastly, TB-PRACTECAL (NCT02589782) assesses shorter regimens containing bedaquiline and pretomanid; its phase 3 component examines the $\mathrm{BPaL}$ regimen supplemented by moxifloxacin. It has recently been stopped early based on promising, unpublished interim results in the experimental arm. endTB prioritizes the combination of bedaquiline and delamanid, in order to maximize the information available to clinicians and policymakers. endTB's implementation in a highly heterogeneous MDR-TB patient population-in seven countries in Africa, Eastern Europe, Asia, and the Americas-will result in evidence that is generalizable to most MDR-TB patients, including HIV-positive patients and adolescents. Such a strong evidence base is most informative for global policy and has been shown to lead to better country uptake [61]. This would result in wider-spread adoption of injectable-sparing, shorter regimens that likely improve patient compliance and simplify the logistical burden on health care systems. These changes can also make treatment accessible to a much higher proportion of patients with drug-resistant TB.

Like Opti-Q, STREAM, STREAM II, and TBPRACTECAL, endTB employs the gold standard of the randomized, concurrently, controlled trial. Several other design decisions were made to "future-proof" the trial despite the established lengthy time required to enrol trial participants for DR-TB [62]. These decisions maximize efficiency and will facilitate endTB's relevance at the time results become available. First, compared to sequential trials, the inclusion of five concurrent experimental arms reduces substantially the sample size and time required to evaluate each of the experimental arms. Second, deployment of Bayesian response-adaptive randomization further reduces the sample size (and time required) by approximately $20 \%$ compared to fixed randomization [33]. Limitations of this approach include the resources required, reliance on an unvalidated interim endpoint to inform adaptation, and possible bias introduced by changes in the study population over time. These limitations have been discussed elsewhere [33]. Third, endTB has allowed the control regimen to keep pace with changing global (and local) policy guidance, protecting its relevance at the end of the study. endTB was among the first trials to allow the use of bedaquiline and delamanid in the comparator. While TB-PRACTECAL also includes this innovation, STREAM did not. When WHO endorsed for the first time the shorter regimen for use in a subset of patients with DR-TB [63], endTB amended the protocol and 
other study documentation to permit the use of the WHO-endorsed shorter regimen in the control arm. Similarly, the WHO recommendation to remove the injectable from conventional (and shorter) regimens [4] was also adopted for endTB control arm regimens. In the Nix-TB trial, the study included no concurrent control [57]. Instead, outcomes in the cohort receiving the $\mathrm{BPaL}$ regimen were compared to those in a historical cohort. The comparator excluded patients who received drugs included in the Nix regimen despite their common inclusion in standard-of-care treatment contemporaneous to the Nix trial [64]. In the case of the STREAM 1 trial, although non-inferiority was established, it was in comparison to standard of care that had become obsolete during the trial. In the endTB trial, the planned comparison to an evolving standard of care does complicate interpretation relative to an ideal situation in which all control-arm participants received the standard of care in force at trial's end. For the sample sizes and length of follow-up currently required for phase 3 trials of drugresistant $\mathrm{TB}$ treatment, and the current dynamic nature of treatment recommendations, this ideal is unlikely to be realized. However, endTB's approach permits the possibility of post hoc comparisons between experimental arms and different compositions of control arms and enhances the chances of meaningful results at the time of study completion. Fourth, endTB is also powered for the improved treatment response expected with recent changes to the standard of care as evidenced by the control arms in the STREAM 1 and delamanid phase 3 trials [45]. endTB sample size simulations accounted for a smaller expected difference between experimental and control arms than that anticipated in the delamanid phase 3 trial. The surprisingly small difference between treatment responses in the control and experimental arms may have been a factor in the "negative" results of the pivotal trial of delamanid [45]. Although a modest improvement in treatment response would not have been considered clinically meaningful in a comparison of two longer, injectable-containing regimens, such a result in one or more shorter, all-oral endTB regimens could be transformative. Fifth, the "hybrid" follow-up approach adopted by endTB balances the objectives of producing results as rapidly as possible while ensuring the ability to detect the majority of relapse cases in the experimental arms. This approach, supported by a reanalysis of relapse timing in trials of shorter TB therapy, was first proposed by Nunn and colleagues [65] as a way to dramatically reduce time to study results with minimal impact on relapse detection. In the endTB trial all participants assigned to experimental arms will have more than 8 months of post-treatment follow-up and $90 \%$ are expected to have more than 12 months of posttreatment observation.
endTB results will not inform treatment of FQresistant TB. endTB ensures the use of $\mathrm{FQ}$ in patients who may be able to benefit from it. And, the endTB consortium has embarked on a second, complementary clinical trial, "Evaluating newly approved drugs in combination regimens for multidrug-resistant tuberculosis with fluoroquinolone resistance" (endTB-Q; NCT03896685), examining fluoroquinolone-sparing regimens only in patients who are not likely to benefit from that drug class. endTB-Q represents, to date, the only randomized, concurrently controlled trial with adequate power to detect treatment effects in patients with fluoroquinolone-resistant TB.

Change in policy and practice around treatment of drug-resistant TB has historically been slow. In the absence of randomized, internally, and concurrently controlled trials, the urgent need for improved treatments, however, has understandably driven recent acceptance of evidence from observational and uncontrolled studies. The endTB trial will close a critical gap by providing high-quality evidence around the safety and efficacy of five injectable-sparing, 9-month multidrug regimens for the treatment of MDR/RR TB.

\section{Trial status}

endTB recruitment began in the first site in February 2017; with expansion to additional sites, enrolment is expected to be complete by mid- 2021 .

\section{Abbreviations}

DSMB: Data Safety Monitoring Board; GCP: Good Clinical Practice; HMS: Harvard Medical School; ICH: International Conference on

Harmonisation; IRB: Institutional Review Board; IRD: Interactive Research \& Development; ITM: Institute of Tropical Medicine; MDR-TB: Multidrug-resistant tuberculosis; mITT: Modified intent-to-treat; MSF: Médecins Sans Frontières; PIH: Partners In Health; PP: Per protocol; RR: Rifampin-resistant;

TB: Tuberculosis; XDR: Extensively drug-resistant

\section{Acknowledgements}

We are grateful for the commitments made by the study participants and for the ongoing supervision and feedback of this trial provided by these three committees: Scientific Advisory Committee (SAC), Data Safety and Monitoring Board (DSMB), and the Global Tuberculosis Community Advisory Board (TB-CAB).

\section{Roles and responsibilities $\{5 \mathrm{~d}\}$}

The trial is sponsored by Médecins Sans Frontières (contact: Dr. Francis Varaine: francis.varaine@paris.msf.org). The sponsor is involved in all trial activities, including study design, data collection and analysis, and writing/ submission of the report. The sponsor has ultimate authority over these activities. The sponsor is responsible for (delegation of) communicating protocol modifications to investigators, participants, and registries after regulatory and ethics approvals. The design and implementation of the trial is supported and overseen by the following external, independent committees: a Data Safety Monitoring Board, a Scientific Advisory Committee, and the Global Tuberculosis Community Advisory Board (TB$(A B)$.

\section{Disclaimer}

The contents of this manuscript are solely the responsibility of the authors and do not necessarily represent the official views of the institutions with which the authors are affiliated. 


\section{Authors' contributions $\{5 \mathrm{a}\}$}

$L G$, FV, and CM wrote the first draft of the manuscript. All authors critically revised the manuscript and gave final approval of the current version to be published.

\section{Funding}

The trial and the preparation of this manuscript are funded by a grant provided by Unitaid. The funding source had no role in the study design; in the collection, management, analysis, or interpretation of data; in the writing of the report; or in the decision to submit the report for publication. GEV received funding from the National Institute of Allergy and Infectious Diseases at the US National Institutes of Health (grant numbers K08 Al141740, L30 Al120170, and P30 Al060354), the Dr. Lynne Reid/Drs. Eleanor and Miles Shore Fellowship at Harvard Medical School, the Burke Global Health Fellowship at the Harvard Global Health Institute, and the Harvard University Center for AIDS Research.

\section{Availability of data and materials}

Not applicable.

\section{Declarations}

\section{Ethics approval and consent to participate $\{24\}\{25\}\{32\}$}

Ethics approval for the study protocol and informed consent materials was granted before the study start from the following IRBs: Médecins sans Frontières Ethics Review Board, Harvard Medical School Institutional Review Board, Interactive Research and Development Institutional Review Board, Institute of Tropical Medicine Institutional Review Board, and IRBs/Ethics Committees in all countries in which the study is implemented. A sample study informed consent form is included as Supplement 2. Any amendments to the protocol or consent materials are reviewed and approved by central IRBs (MSF, HMS) before they are submitted to local authorities and local IRBs for approval.

\section{Consent for publication}

Not applicable.

\section{Competing interests $\{28\}$}

The endTB Consortium coordinated donations of delamanid (Otsuka Pharmaceutical) and bedaquiline (Janssen) to be used for treatment by some of the patients included in the endTB Observational Study. Authors report the following potential conflicts of interest: None other than the donations disclosed above.

\section{Author details}

${ }^{1}$ Médecins Sans Frontières, Paris, France. ${ }^{2}$ Sorbonne Université, INSERM, U1135, Centre d'Immunologie Et Des Maladies Infectieuses, Paris, France. ${ }^{3}$ Assistance Publique Hôpitaux de Paris, Groupe Hospitalier Universitaire Sorbonne Université, Hôpital Pitié-Salpêtrière, Centre National De Référence Des Mycobactéries Et De La Résistance Des Mycobactéries Aux Antituberculeux, Paris, France. Institute of Tropical Medicine, Antwerp, Belgium. ${ }^{5}$ Epicentre, Paris, France. ${ }^{6}$ Partners In Health, Astana, Kazakhstan. ${ }^{7}$ National Scientific Center of Phthisiopulmonology, Almaty, Kazakhstan. ${ }^{8}$ Institut de Recherche pour le Développement/INSERM U1175/UMI233/ Université de Montpellier, Montpellier, France. ${ }^{9}$ Médecins Sans Frontières, Toronto, Ontario, Canada. ${ }^{10}$ Department of Global Health and Social Medicine, Harvard Medical School, Boston, MA, USA. ${ }^{11}$ Centre for Infectious Disease Epidemiology and Research, School of Public Health and Medicine, Faculty of Health Sciences, University of Cape Town, Cape Town, South Africa. ${ }^{12}$ Southern Africa Medical Unit, Médecins Sans Frontières, Cape Town, South Africa. ${ }^{13}$ Interactive Research and Development, Karachi, Pakistan. ${ }^{14}$ Socios En Salud-Sucursal Peru, Lima, Peru. ${ }^{15}$ Assistance Publique Hôpitaux de Paris, Unité de Recherche Clinique, Hôpital Pitié-Salpêtrière, Paris, France. ${ }^{16}$ Division of Clinical Pharmacology, Department of Medicine, University of Cape Town, Cape Town, South Africa. ${ }^{17}$ Wellcome Centre for Infectious Diseases Research in Africa, Institute of Infectious Disease and Molecular Medicine, University of Cape Town, Cape Town, South Africa. ${ }^{18}$ Abiomed, Inc., Danvers, MA, USA. ${ }^{19}$ Social \& Scientific Systems-DLH, Silver Spring, MD, USA. ${ }^{20}$ Partners In Health, Maseru, Lesotho. ${ }^{21}$ Epidemiology and Communicable Diseases Division, Indian Council of Medical Research, Pune, India. ${ }^{22}$ Indian Council of Medical Research - National AIDS Research
Institute, Pune, India. ${ }^{23}$ University of San Francisco Center for Tuberculosis, San Francisco, CA, USA. ${ }^{24}$ Partners In Health, Boston, MA, USA. ${ }^{25}$ Division of Global Health Equity, Brigham and Women's Hospital, Boston, MA, USA. ${ }^{26}$ Department of Infectious Diseases, Indus Hospital, Karachi, Pakistan.

${ }^{27}$ Dana-Farber Cancer Institute, Boston, MA, USA. ${ }^{28}$ Harvard T.H. Chan School of Public Health, Boston, MA, USA. ${ }^{29}$ Division of Infectious Diseases, Brigham and Women's Hospital, Boston, MA, USA. ${ }^{30}$ Wellcome Centre for Infectious Diseases Research in Africa, Department of Medicine, University of Cape Town, Cape Town, South Africa. ${ }^{31}$ Division of Infectious Diseases and HIV Medicine, Department of Medicine, Groote Schuur Hospital and University of Cape Town, Cape Town, South Africa. ${ }^{32}$ Harvard Medical School, Boston, MA, USA. ${ }^{33}$ Beth Israel Deaconess Medical Center, Boston, MA, USA.

Received: 8 March 2021 Accepted: 27 July 2021

Published online: 25 September 2021

\section{References}

1. WHO. Global tuberculosis report 2015. Geneva: World Health Organization; 2015.

2. Siqueira HR, Freitas FA, Oliveira DN, Barreto AM, Dalcolmo MP, Albano RM. Clinical evolution of a group of patients with multidrug-resistant TB treated at a referral center in the city of Rio de Janeiro, Brazil. J Bras Pneumol. 2009. https://doi.org/10.1590/s1806-37132009000100008.

3. Frank M, Adamashvili N, Lomtadze N, Kokhreidze E, Avaliani Z, Kempker RR, Blumberg HM. Long-term Follow-up Reveals High Posttreatment Mortality Rate Among Patients With Extensively Drug-Resistant Tuberculosis in the Country of Georgia. Open Forum Infect Dis. 2019. https://doi.org/10.1093/ ofid/ofz152.

4. WHO. WHO consolidated guidelines on tuberculosis. Module 4: treatment - drugresistant tuberculosis treatment. Geneva: World Health Organization; 2020.

5. Bonnet M, Bastard M, du Cros P, Khamraev A, Kimenye K, Khurkhumal S, et al. Identification of patients who could benefit from bedaquiline or delamanid: a multisite MDR-TB cohort study. Int J Tuberc Lung Dis. 2016. https://doi.org/10.5588/ijtld.15.0962.

6. WHO. Global tuberculosis report 2020. Geneva: World Health Organization; 2020.

7. Lan Z, Ahmad N, Baghaei P, Barkane L, Benedetti A, Brode SK, et al. Drugassociated adverse events in the treatment of multidrug-resistant tuberculosis: an individual patient data meta-analysis. Lancet Respir Med. 2020. https://doi.org/10.1016/S2213-2600(20)30047-3.

8. Diacon AH, Pym A, Grobusch MP, de los Rios JM, Gotuzzo E, Vasilyeva I, et al. Multidrug-resistant tuberculosis and culture conversion with Bedaquiline. N Engl J Med. 2014;371(8):723-32. https://doi.org/10.1056/ NEJMoa1313865.

9. Gler M, Skrpconoka V, Sanchez-Garavito E. Delamanid for multidrug-resistant pulmonary tuberculosis. N Engl J Med. 2012;366:2151-6.

10. Bloss $\mathrm{E}$, et al. Adverse events related to multidrug-resistant tuberculosis treatment, Latvia, 2000-2004. Int J Tuberc Lung Dis. 2010;14:275-81.

11. Shin SS, Pasechnikov AD, Gelmanova IY, Peremitin GG, Strelis AK, Mishustin $S$, et al. Treatment outcomes in an integrated civilian and prison MDR-TB treatment program in Russia. Int J Tuberc Lung Dis. 2006;10(4):402-8.

12. Wu S, Zhang $Y$, Sun F, Chen M, Zhou L, Wang N, Zhan S. Adverse Events Associated With the Treatment of Multidrug-Resistant Tuberculosis: A Systematic Review and Meta-analysis. Am J Ther. 2016. https://doi.org/10.1 097/01.mjt.0000433951.09030.5a.

13. Nathanson E, Gupta R, Huamani P, Leimane V, Pasechnikov AD, Tupasi TE, et al. Adverse events in the treatment of multidrug-resistant tuberculosis: results from the DOTS-Plus initiative. Int J Tuberc Lung Dis. 2004;8(11):1382-4.

14. Sanchez-Padilla E, Marquer C, Kalon S, Qayyum S, Hayrapetyan A, Varaine F, et al. Reasons for defaulting from drug-resistant tuberculosis treatment in Armenia: a quantitative and qualitative study. Int J Tuberc Lung Dis. 2014. https://doi.org/10.5588/ijtld.13.0369.

15. Shean K, Streicher E, Pieterson E, Symons G, van Zyl Smit R, Theron G, et al. Drug-associated adverse events and their relationship with outcomes in patients receiving treatment for extensively drug-resistant tuberculosis in South Africa. PLoS One. 2013. https://doi.org/10.1371/annotation/644591a88ae6-450e-974e-1cd1f08f52c7.

16. Nunn AJ, Phillips PPJ, Meredith SK, Chiang CY, Conradie F, Dalai D, et al. A trial of a shorter regimen for rifampin-resistant tuberculosis. N Engl J Med. 2019. https://doi.org/10.1056/NEJMoa1811867. 
17. Aung KJ, Van Deun A, Declerca E, Sarker MR, Das PK, Hossain MA, Rieder HL. Successful '9-month Bangladesh regimen' for multidrug-resistant tuberculosis among over 500 consecutive patients. Int J Tuberc Lung Dis. 2014. https://doi.org/10.5588/ijtld.14.0100.

18. Lange C, Duarte R, Jachym M, Guenther G, Guglielmetti L. Limited benefit of the new shorter multidrug-resistant tuberculosis regimen in Europe. Am J Respir Crit Care Med. 2016. https://doi.org/10.1164/rccm.201606-1097LE.

19. Sotgiu G, Tiberi S, D'Ambrosio L, Centis R, Alffenaar JW, Caminero JA, et al. Faster for less: the new "shorter" regimen for multidrug-resistant tuberculosis. Eur Respir J. 2016. https://doi.org/10.1183/13993003.01249-2016.

20. Skripconoka V, Danilovits M, Pehme L, Tomson T, Skenders G, Kummik T, et al. Delamanid improves outcomes and reduces mortality in multidrug-resistant tuberculosis. Eur Respir J. 2013. https://doi.org/10.1183/09031936.00125812.

21. Pym AS, Diacon AH, Tang SJ, Conradie F, Danilovits M, Chuchottaworn C, et al. TMC207-C209 Study Group. Bedaquiline in the treatment of multidrug- and extensively drug-resistant tuberculosis. Eur Respir J. 2016. https://doi.org/10.1183/13993003.00724-2015.

22. Sotgiu G, Centis R, D'Ambrosio L, Alffenaar JWC, Anger HA, Caminero JA, et al. Efficacy, safety and tolerability of linezolid containing regimens in treating MDR-TB and XDR-TB: systematic review and meta-analysis. Eur Respir J. 2012. https://doi.org/10.1183/09031936.00022912.

23. Cox H, Ford N. Linezolid for the treatment of complicated drug-resistant tuberculosis: a systematic review and meta-analysis. Int J Tuberc Lung Dis. 2012. https://doi.org/10.5588/ijtld.11.0451.

24. Lee M, Lee J, Carroll MW, Choi H, Min S, Song T, et al. Linezolid for treatment of chronic extensively drug-resistant tuberculosis. N Engl I Med. 2012. https://doi.org/10.1056/NEJMoa1201964.

25. Lee M, Cho S-N, Barry CE. Linezolid for XDR-TB - final study outcomes. N Engl J Med. 2015;373(3):290-1. https://doi.org/10.1056/ NEJMc1500286.

26. Tang S, Yao L, Hao X. Efficacy, safety and tolerability of linezolid for the treatment of XDR-TB: a study in China. Eur Respir J. 2015. https://doi.org/1 $0.1183 / 09031936.00035114$

27. Padayatchi N, Gopal M, Naidoo R, Werner L, Naidoo K, Master I, et al. Clofazimine in the treatment of extensively drug-resistant tuberculosis with HIV coinfection in South Africa: a retrospective cohort study. J Antimicrob Chemother. 2014. https://doi.org/10.1093/jac/dku235.

28. Tang S, Yao L, Hao X, Liu Y, Zeng L, Liu G, Li M, Li F, Wu M, Zhu Y, Sun H, Gu J, Wang X, Zhang Z. Clofazimine for the treatment of multidrug-resistant tuberculosis: prospective, multicenter, randomized controlled study in China. Clin Infect Dis. 2015. https://doi.org/10.1093/cid/civ027.

29. Williams K, Minkowski A, Amoabeng O, Peloquin CA, Taylor D, Andries K, et al. Sterilizing activities of novel combinations lacking first- and secondline drugs in a murine model of tuberculosis. Antimicrob Agents Chemother. 2012. https://doi.org/10.1128/AAC.00384-12.

30. Ibrahim M, Andries K, Lounis N, Chauffour A, Truffot-Pernot C, Jarlier V, et al. Synergistic activity of R207910 combined with pyrazinamide against murine tuberculosis. Antimicrob Agents Chemother. 2007. https://doi.org/10.112 8/AAC.00898-06

31. Tasneen R, Li SY, Peloquin CA, Taylor D, Williams KN, Andries K, et al. Sterilizing activity of novel TMC207- and PA-824-containing regimens in a murine model of tuberculosis. Antimicrob Agents Chemother. 2011. https:// doi.org/10.1128/AAC.05293-11.

32. Brigden G, Nyang'wa BT, du Cros P, Varaine F, Hughes J, Rich M, et al. Principles for designing future regimens for multidrug-resistant tuberculosis. Bull World Health Organ. 2014;92(1):68-74. https://doi.org/10.2471/BLT.13.122028.

33. Cellamare M, Milstein M, Ventz S, Baudin E, Trippa L, Mitnick CD. Bayesian adaptive randomization in a clinical trial to identify new regimens for MDRTB: the endTB trial. Int J Tuberc Lung Dis. 2016;20(12):8-12. https://doi.org/1 $0.5588 /$ ijtld. 16.0066 .

34. Cellamare M, Ventz S, Baudin E, Mitnick CD, Trippa L. A Bayesian responseadaptive trial in tuberculosis: the endTB trial. Clin Trials I Soc Clin Trials. 2017;14(1):17-28. https://doi.org/10.1177/1740774516665090.

35. Mccune RM, Mcdermott W, Tompsett R. The fate of mycobacterium tuberculosis in mouse tissues as determined by the microbial enumeration technique. II. The conversion of tuberculous infection to the latent state by the administration of pyrazinamide and a companion drug. J Exp Med. 1956;104:763-802.

36. Mitchison DA. The diagnosis and therapy of tuberculosis during the past 100 years. Am J Respir Crit Care Med. 2005;171(7):699-706. https://doi.org/1 0.1164/rccm.200411-1603OE.
37. Chang KC, Yew WW, Zhang Y. Pyrazinamide susceptibility testing in mycobacterium tuberculosis : a systematic review with meta-analyses. Antimicrob Agents Chemother. 2011;55(10):4499-505. https://doi.org/10.112 8/AAC.00630-11.

38. Ahmad Z, Tyagi S, Minkowski A, Peloquin CA, Grosset JH, Nuermberger EL. Contribution of moxifloxacin or levofloxacin in second-line regimens with or without continuation of pyrazinamide in murine tuberculosis. Am J Respir Crit Care Med. 2013;188(1):97-102. https://doi.org/10.1164/rccm.2 01212-23280C.

39. Jacobson KR, Tierney DB, Jeon CY, Mitnick CD, Murray MB. Treatment outcomes among patients with extensively drug-resistant tuberculosis: systematic review and meta-analysis. Clin Infect Dis. 2010;02114:6-14.

40. Falzon D, Jaramillo E, Schunemann HJ, Arentz M, Bauer M, Bayona J, et al. WHO guidelines for the programmatic management of drug-resistant tuberculosis: 2011 update. Eur Respir J. 2011;38(3):516-28. https://doi.org/1 0.1183/09031936.00073611.

41. Fox W, Ellard GA, Mitchison DA. Studies on the treatment of tuberculosis undertaken by the British Medical Research Council tuberculosis units, 1946-1986, with relevant subsequent publications. Int J Tuberc Lung Dis. 1999:3(10 Suppl 2):S231-79.

42. WHO. Guidelines for the Programmatic Management of Multidrug-Resistant Tuberculosis. Geneva: World Health Organization; 2011.

43. WHO. Rapid communication: key changes to treatment of drug-resistant tuberculosis. Geneva: World Health Organization; 2019.

44. National Cancer Institute. Common terminology criteria for adverse events, version 4.0. http://evs.nci.nih.gov/ftp1/CTCAE. Accessed 15 Sept 2017.

45. von Groote-Bidlingmaier F, Patientia R, Sanchez E, Balanag V Jr, Ticona E, Segura P, et al. Efficacy and safety of delamanid in combination with an optimised background regimen for treatment of multidrug-resistant tuberculosis: a multicentre, randomised, double-blind, placebo-controlled, parallel group phase 3 trial. Lancet Respir Med. 2019;7(3):249-59. https://doi. org/10.1016/S2213-2600(18)30426-0.

46. Van Deun A, et al. Short, highly effective, and inexpensive standardized treatment of multidrug-resistant tuberculosis. Am J Respir Crit Care Med. 2010;182(5):684-92. https://doi.org/10.1164/rccm.201001-00770C.

47. Aung KJM, van Deun A, Declercq E, Sarker MR, Das PK, Hossain MA, et al. Successful '9-month Bangladesh regimen' for multidrug-resistant tuberculosis among over 500 consecutive patients. Int J Tuberc Lung Dis Off J Int Union Tuberc Lung Dis. 2014;18(10):1180-7. https://doi.org/10. 5588/ijtld.14.0100.

48. Piubello A, Harouna SH, Souleymane MB, Boukary I, Morou S, Daouda M, et al. High cure rate with standardised short-course multidrug-resistant tuberculosis treatment in Niger: no relapses. Int J Tuberc Lung Dis. 2014; 18(10):1188-94. https://doi.org/10.5588/ijtld.13.0075.

49. Kuaban C, Noeske J, Rieder HL, Aït-Khaled N, Abena Foe JL, Trébucq A. High effectiveness of a 12-month regimen for MDR-TB patients in Cameroon. Int J Tuberc Lung Dis. 2015;19(5):517-24. https://doi.org/10.5588/ijtld.14.0535.

50. Kendall EA, Fofana MO, Dowdy DW. Burden of transmitted multidrug resistance in epidemics of tuberculosis: a transmission modelling analysis. Lancet Respir Med. 2015;3(12):963-72. https:/doi.org/10.1016/52213-2600(15)00458-0.

51. Sineke T, Evans D, Schnippel K, van Aswegen H, Berhanu R, Musakwa N, et al. The impact of adverse events on health-related quality of life among patients receiving treatment for drug-resistant tuberculosis in Johannesburg, South Africa. Health Qual Life Outcomes. 2019. https://doi. org/10.1186/s12955-019-1155-4.

52. Abidi S, Achar J, Assao Neino MM, Bang D, Benedetti A, Brode S, et al. Standardised shorter regimens versus individualised longer regimens for rifampin- or multidrug-resistant tuberculosis. Eur Respir J. 2020;55(3): 1901467. https://doi.org/10.1183/13993003.01467-2019.

53. Zhao Y, Fox T, Manning K, Stewart A, Tiffin N, Khomo N, et al. Improved treatment outcomes with bedaquiline when substituted for second-line injectable agents in multidrug-resistant tuberculosis: a retrospective cohort study. Clin Infect Dis Off Publ Infect Dis Soc Am. 2019;68(9):1522-9. https:// doi.org/10.1093/cid/ciy727.

54. Ndjeka N, Schnippel K, Master I, Meintjes G, Maartens G, Romero R, et al. High treatment success rate for multidrug-resistant and extensively drug-resistant tuberculosis using a bedaquiline-containing treatment regimen. Eur Respir J. 2018;52(6):1801528. https://doi.org/1 0.1183/13993003.01528-2018.

55. Peloquin CA, Phillips PPJ, Mitnick CD, Eisenach K, Patientia RF, Lecca L, et al. Increased doses lead to higher drug exposures oflevofloxacin for treatment 
of tuberculosis. Antimicrob Agents Chemother. 2018. https://doi.org/10.112 8/AAC.00770-18.

56. Franke MF, Khan P, Hewison C, Khan U, Huerga H, Seung KJ, et al. Culture conversion in patients treated with bedaquiline and/or delamanid: a prospective multi-country study. Am J Respir Crit Care Med. 2020;203(1): 111-9. https://doi.org/10.1164/rccm.202001-01350C.

57. Conradie F, Diacon AH, Ngubane N, Howell P, Everitt D, Crook AM, et al. Treatment of highly drug-resistant pulmonary tuberculosis. N Engl J Med. 2020;382(10):893-902. https://doi.org/10.1056/NEJMoa1901814.

58. McKenna L, Furin J. Are pretomanid-containing regimens for tuberculosis a victory or a victory narrative? Lancet Respir Med. 2019;7(12):999-1000. https://doi.org/10.1016/S2213-2600(19)30363-7.

59. Guglielmetti L, Low M, McKenna L. Challenges in TB regimen development: preserving evidentiary standards for regulatory decisions and policymaking. Expert Rev Anti-Infect Ther. 2020;18(8):701-4. https://doi.org/10.1080/14 787210.2020 .1756776$.

60. Global TB Community Advisory Board. Research, regulatory, and access considerations regarding pretomanid. 2019. https://www.tbonline.info/ media/uploads/documents/tb_cab_pretomanid_nda_considerations_final_ 05.14.19.pdf. Accessed 4 Aug 2021.

61. Nasser SMU, Cooke G, Kranzer K, Norris SL, Olliaro P, Ford N. Strength of recommendations in WHO guidelines using GRADE was associated with uptake in national policy. J Clin Epidemiol. 2015;68(6):703-7. https://doi. org/10.1016/j.jclinepi.2014.11.006.

62. Phillips PPJ, Mitnick CD, Neaton JD, Nahid P, Lienhardt C, Nunn AJ. Keeping phase III tuberculosis trials relevant: adapting to a rapidly changing landscape. PLoS Med. 2019;16(3):e1002767. https:/doi.org/10.1371/journal. pmed. 1002767.

63. WHO. WHO treatment guidelines for drug-resistant tuberculosis, 2016 update. Geneva: World Health Organization; 2016.

64. FDA. Drug Approval Package: Pretomanid. 2019. https://www.accessdata.fda. gov/drugsatfda_docs/nda/2019/2128620rig15000TOC.cfm. Accessed 5 Mar 2020.

65. Nunn AJ, Phillips P, Mitchison D. Timing of relapse in short-course chemotherapy trials for tuberculosis. Int J Tuberc Lung Dis. 2010;14(2):241-2.

\section{Publisher's Note}

Springer Nature remains neutral with regard to jurisdictional claims in published maps and institutional affiliations.

Ready to submit your research? Choose BMC and benefit from:

- fast, convenient online submission

- thorough peer review by experienced researchers in your field

- rapid publication on acceptance

- support for research data, including large and complex data types

- gold Open Access which fosters wider collaboration and increased citations

- maximum visibility for your research: over $100 \mathrm{M}$ website views per year

At BMC, research is always in progress.

Learn more biomedcentral.com/submissions 